\title{
THE EQUIVALENCE OF FIBER SPACES AND BUNDLES 1
}

\author{
BY EDWARD FADELL
}

Communicated by Hans Samelson, October 8, 1959

1. Introduction. The objective of this paper is to verify the conjecture made in [2] that every Hurewicz fibration [3] over a polyhedral base is fiber homotopy equivalent to a Steenrod fiber bundle [6]. The result relies heavily on Milnor's universal bundle construction [4] and the following extension [2] of a theorem of A. Dold [1].

Theorem. If $\left\{E_{1}, p_{1}, X\right\}$ and $\left\{E_{2}, p_{2}, X\right\}$ are Hurewicz fibrations over a connected $C W$-complex $X$ and if $f: E_{1} \rightarrow E_{2}$ is a fiber-preserving map such that $f$ restricted to some fiber is a homotopy equivalence, then $f$ is a fiber homotopy equivalence.

2. The associated bundle. Let $\pi: E \rightarrow X$ denote a map, where $X$ is a connected, locally finite polyhedron. Furthermore following Milnor's notation in [4], let $\tilde{S}, \tilde{E}, \widetilde{G}$ denote, respectively, the simplicial paths in $X$, the simplicial paths emanating from a fixed vertex $v_{0}$ and the simplicial loops at $v_{0}$. If $\alpha=\left[x_{n}, \cdots, x_{0}\right]$ is a simplicial path in $X$ we will find it convenient to set $\alpha(0)=x_{0}, \alpha(1)=x_{n}$. Now, define

$$
\Omega_{\pi}=\{(e, \alpha) \in E \times \widetilde{S} \mid \pi(e)=\alpha(0)\}
$$

and a map $\xi: \Omega_{\pi} \rightarrow X$ by

$$
\xi(e, \alpha)=\alpha(1)
$$

Furthermore, let

$$
A=\xi^{-1}\left(v_{0}\right)=\left\{(e, \alpha) \mid \pi(e)=\alpha(0), \alpha(1)=v_{0}\right\} .
$$

Lemma. $\left\{\Omega_{\pi}, \xi, X, A, \widetilde{G}\right\}$ is a Steenrod fiber bundle.

PROOF. Since the proof is entirely analogous to Milnor's proof [4] that $\tilde{E}$ is a bundle over $X$, we content ourselves with a brief outline. The action $\mu: \widetilde{G} \times A \rightarrow A$ is defined as follows:

$$
\mu[g,(e, \alpha)]=(e, g \alpha) .
$$

Now, let $v_{j}$ denote a vertex in $X$ and $V_{j}$ the star neighborhood of $v_{j}$. The coordinate functions

$$
\phi_{j}: V_{j} \times A \rightarrow \xi^{-1}\left(V_{j}\right)
$$

are defined by

1 This research was supported, in part, by the Wisconsin Alumni Research Foundation. 


$$
\phi_{j}(x,(e, \alpha))=\left(e,\left[x, v_{j}\right] e_{j} \alpha\right)
$$

where $e_{j}$ is a fixed simplicial path from $v_{0}$ to $v_{j}$. We leave the remaining details to the reader.

Now, define $f: E \rightarrow \Omega_{\pi}$ by

$$
f(e)=(e,[\pi(e), \pi(e)]) .
$$

The following diagram is easily seen commutative:

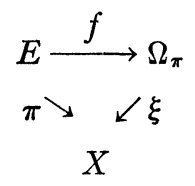

3. The equivalence theorem. Let $\pi: E \rightarrow X, f: E \rightarrow \Omega_{\pi}$ be as in $\S 2$.

Theorem. If $\{E, \pi, X\}$ is a Hurewicz fibration, then $f$ is a fiber homotopy equivalence.

Proof. Let $F=\pi^{-1}\left(v_{0}\right)$ denote the fiber in $E$ over $v_{0}$. Then, in view of the theorem mentioned in the introduction, it suffices to show that $f^{\prime}=f \mid F: F \rightarrow A$ is a homotopy equivalence.

Let $\bar{X}$ denote the space of ordinary paths in $X$ ending at $v_{0}$ and let $\eta: \bar{X} \rightarrow X$ denote the fiber map given by $\eta(\alpha)=\alpha(0)$. Furthermore, let $\bar{E}$ denote the space of simplicial paths $\left[x_{n}, \cdots, x_{0}\right]$ on $X$ such that $x_{n}=v_{0}$. Then, since $\bar{E}$ is homeomorphic to $\tilde{E}$ under the correspondence $\left[x_{n}, \cdots, x_{0}\right] \leftrightarrow\left[x_{0}, \cdots, x_{n}\right], \bar{E}$ is a fiber bundle over $X$ with fiber map $p: \bar{E} \rightarrow X$, given by $p\left(\left[x_{n}, \cdots, x_{0}\right]\right)=x_{0}$ and fiber $\widetilde{G}$. Consider then the fiber-preserving map $\bar{h}$

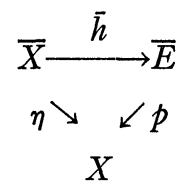

defined as follows: Let $\bar{\lambda}$ denote a regular lifting function for $\{\bar{E}, p, X\}$ and if $\alpha \in \bar{X}$, set $\bar{\alpha}(t)=\alpha(1-t), 0 \leqq t \leqq 1$. Finally, define

$$
\bar{h}(\alpha)=\bar{\lambda}\left(\left[v_{0}, v_{0}\right], \bar{\alpha}\right)(1) \text {. }
$$

Now, $\bar{X}$ and $\bar{E}$ are contractible, $\eta^{-1}\left(v_{0}\right)=\Omega(X)$, the space of ordinary loops on $X$, is dominated by a $C W$-complex and $\widetilde{G}$ is a $C W$-complex. Therefore $\bar{h}$ restricted to $\Omega(X)$ is a homotopy equivalence and we may conclude that $\bar{h}$ is a fiber homotopy equivalence. Thus $\bar{h}$ possesses a fiber homotopy inverse $h$. If $\tilde{v}_{0} \in \bar{X}$ is the constant path and $\left[v_{0}, v_{0}\right] \in \widetilde{G}$ is the identity in $\widetilde{G}$, then $\bar{h}\left(\tilde{v}_{0}\right)=\left[v_{0}, v_{0}\right]$ and $h$ may be 
chosen so that $h\left(\left[v_{0}, v_{0}\right]\right)=\tilde{v}_{0}$. We employ $h$ and $\bar{h}$ to define an auxiliary map $\chi: A \rightarrow A$ as follows. Define

$$
\chi(e, \alpha)=(e, \bar{h} h(\alpha)) .
$$

Since $\bar{h} h$ is fiber homotopic to the identity map $\bar{E} \rightarrow \bar{E}, \chi \sim 1: A \rightarrow A$.

Next, we define a homotopy $H: A \times I \rightarrow A$. If $\omega$ is an ordinary path in $X$ and $0 \leqq s, t \leqq 1$, set

$$
\omega_{s}(t)=\omega(s t)
$$

and

$$
\omega^{*}(t)=\omega(s+t-s t) .
$$

Then, define, for $0 \leqq s \leqq 1$,

$$
H((e, \alpha), s)=\left\{\lambda\left(e,[h(\alpha)]_{s}\right)(1), \bar{h}\left([h(\alpha)]^{s}\right)\right\}
$$

where $\lambda$ is a regular lifting function for $\{E, \pi, B\}$. Note that $\pi \lambda\left(e,[h(\alpha)]_{s}\right)(1)=h(\alpha)(s)=\bar{h}\left([h(\alpha)]^{s}\right)(0)$ since $\bar{h}$ preserves end points and $[h(\alpha)]^{s}(0)=h(\alpha)(s)$. Also $\bar{h}\left([h(\alpha)]^{s}\right)(1)=v_{0}$ for the same reason. Thus, $H((e, \alpha), s) \in A$. Furthermore,

$$
\begin{aligned}
& H_{0}(e, \alpha)=(e, \bar{h} h(\alpha))=\chi(e, \alpha), \\
& H_{1}(e, \alpha)=\left\{\lambda(e, h(\alpha))(1),\left[v_{0}, v_{0}\right]\right\}
\end{aligned}
$$

where $\left[v_{0}, v_{0}\right]$ is the identity in $\tilde{G}$.

Finally, we define the required homotopy inverse for $f^{\prime}: F \rightarrow A$. Set

$$
g(e, \alpha)=\lambda(e, h(\alpha))(1) .
$$

Then, if $y \in F$,

$$
g f^{\prime}(y)=g\left(y,\left[v_{0}, v_{0}\right]\right)=\lambda\left(y, \tilde{v}_{0}\right)(1)=y
$$

and hence $g f^{\prime}=1$. Also, if $(e, \alpha) \in A$,

$$
f^{\prime} g(e, \alpha)=\left(\lambda(e, h(\alpha))(1),\left[v_{0}, v_{0}\right]\right)=H_{1}(e, \alpha) .
$$

Therefore $f^{\prime} g \sim \chi \sim 1$ and $g$ is a homotopy inverse for $f^{\prime}$. This proves the equivalence theorem.

REMARK. It is not difficult to check that $F$ considered as a subset of $A$ is actually a strong deformation retract of $A$.

REMARK. It is quite clear that our main result is false for Serre fibrations [5] since there exist Serre fibrations over the unit interval whose fibers are not of the same homotopy type. Also, it is possible to exhibit examples of Hurewicz fibrations with 0 -connected but not locally contractible base spaces for which our main result is false. 
4. Extensions. The Equivalence Theorem is also valid if the base space $X$ is dominated by a locally finite polyhedron. Thus, our main result can be stated as follows.

THEOREM. Every Hurewicz fibration over a base space dominated by a locally finite polyhedron is fiber homotopy equivalent to a Steenrod fiber bundle.

An interesting application is the following corollary.

Corollary. If $X$ is a connected space dominated by a locally finite polyhedron, then for every integer $n \geqq 1$, there exist $n$-connective Steenrod fiber bundles over $X$.

Proof. One merely applies the above theorem to the $n$-connective Hurewicz fibrations over $X$ given by G. W. Whitehead in [7].

\section{BIBLIOGRAPHY}

1. A. Dold, Über fasernweise Homotopieäquivalenz von Faserräumen, Math. Z. vol. 62 (1955) pp. 111-136.

2. E. Fadell, On fiber homotopy equivalence, to appear in Duke Math. J.

3. W. Hurewicz, On the concept of fiber space, Proc. Nat. Acad. Sci. U.S.A. vol. 41 (1955) pp. 956-961.

4. J. Milnor, Construction of universal bundles, I, Ann. of Math. vol. 63 (1956) pp. 272-284.

5. J. P. Serre, Homologie singulière des espaces fibrés, Ann. of Math. vol. 54 (1951) pp. 425-505.

6. N. Steenrod, The topology of fiber bundles, Princeton, 1951.

7. G. W. Whitehead, Fiber spaces and the Eilenberg homology groups, Proc. Nat. Acad. Sci. U.S.A. vol. 38 (1952) pp. 426-430.

UNIVERSITY OF WISCONSIN 